\title{
Aspectos da interconsulta psiquiátrica em hospital de trauma
}

\author{
Ricardo Schmitt* \\ Roberta Horn Gomes**
}

\section{INTRODUÇÃO}

A inserção da psiquiatria em hospitais gerais não é uma novidade. Na realidade, esboços dessa relação já existiam desde o século XVIII ${ }^{1,2}$. No entanto, foi a partir do século XX, principalmente após a II Guerra Mundial, que esse fenômeno cresceu em importância nos Estados Unidos e na Europa. No Brasil, o surgimento de enfermarias psiquiátricas em hospitais gerais iniciou-se após a década de 1950, mas foi com a discussão da reforma psiquiátrica ${ }^{3}$ que tal evento ganhou força.

Com a chegada de psiquiatras nos hospitais gerais, surgiu um novo campo de atuação: a interconsulta. A literatura é repleta de publicações a respeito dessa nova área de

Este trabalho foi realizado no Centro de Ciências da Saúde, Universidade Comunitária Regional de Chapecó (UNOCHAPECÓ).

* Médico psiquiatra. Mestre em Psiquiatria pela UFRGS. Centro de Ciências da Saúde, UNOCHAPECÓ. Docente do Instituto de Formação em Teoria Psicanalítica, GEPA, Chapecó, SC. Ex-médico contratado do Hospital Cristo Redentor, Porto Alegre, RS.

** Médica psiquiatra. Ex-residente de Psiquiatria do Grupo Hospitalar Conceição. exercício da psiquiatria ${ }^{4,5}$. São diversos os estudos a respeito do conceito, técnicas de diagnóstico, intervenções e epidemiologia da chamada interconsulta psiquiátrica em hospital geral $^{1,2,4,5}$.

A demanda por psiquiatras vem crescendo também em outra área da medicina hospitalar: o hospital de trauma. A doença trauma vem exigindo uma crescente especialização de diversas áreas da Medicina, como cirurgia, ortopedia e neurologia. A psiquiatria, uma vez inserida no hospital geral, precisa adaptar-se também a essas necessidades. No entanto, nessa área específica da interconsulta psiquiátrica, poucos estudos têm sido publicados ${ }^{6}$.

O objetivo deste trabalho foi revisar os principais aspectos da interconsulta psiquiátrica em hospital de trauma. Para isso, o artigo foi estruturado nos seguintes itens: a) revisão da psiquiatria no hospital geral (onde se origina a interconsulta psiquiátrica) e b) psiquiatria no hospital de trauma e seus aspectos particulares.

As fontes bibliográficas foram pesquisadas através de: a) fontes eletrônicas - MEDLINE 
(1993-2003) e LILACS (1993-2003); b) resumos de conferências e anais de congressos; c) revisão de capítulos sobre interconsulta psiquiátrica e psiquiatria em hospital de trauma em livros textos. Os termos usados na busca foram: interconsulta e psiquiatria; psiquiatria e trauma; interconsulta e trauma; hospital e psiquiatria; hospital e trauma.

Foram selecionadas fontes que continham informações sobre os aspectos principais da interconsulta psiquiátrica em hospital geral, que serviram como base para a primeira parte do artigo; assim como fontes que continham trabalhos de interconsulta psiquiátrica em hospital de trauma, com dados epidemiológicos e clínicos dos transtornos psiquiátricos associados ao trauma.

Foram excluídos da revisão aqueles trabalhos que versavam sobre aspectos específicos da interconsulta psiquiátrica em hospital geral que não eram relevantes para 0 objetivo principal do trabalho, e textos sobre trauma que não faziam menção à avaliação das comorbidades e complicações psiquiátricas possivelmente associadas.

\section{DESENVOLVIMENTO}

\section{1) Interconsulta psiquiátrica em hospital geral - Histórico}

No que se refere ao Brasil, a interconsulta vem ganhando destaque e importância principalmente desde a década de 1980. Nesse período, diversos encontros científicos têm contemplado essa questão. Como exemplo, podemos citar os simpósios promovidos pela Escola Paulista de Medicina, congressos da Universidade Federal de São Paulo (USP) e encontros da Associação Brasileira de Psiquiatria (ABP), entre outros.Atualmente, algumas associações de reconhecida importância na área da psiquiatria contam com departamentos ou núcleos de interconsulta psiquiátrica, como, por exemplo, o Departamento de Interconsulta e Psiquiatria de Hospital Geral da ABP e o Núcleo de Interconsulta e Hospital Geral da Sociedade de Psiquiatria do Rio Grande do Sul'1.

A importância da interconsulta aumentou ainda mais com a promulgação da Lei $n^{\circ} 10216$ de 06 de abril de $2001^{3}$, que instituiu a reforma psiquiátrica. Tal lei recomenda a criação de unidades psiquiátricas em hospitais gerais, tornando uma norma a relação já existente entre a psiquiatria e outras especialidades médicas.
Temos, portanto, estabelecido um contexto propício para a integração de diversas áreas da Medicina, vindo ao encontro de uma abordagem biopsicossocial do indivíduo doente. Tal realidade exige, por outro lado, o reconhecimento, por parte dos psiquiatras, de que pertencem a uma especialidade médica. A interconsulta tem sido fundamental no retorno da psiquiatria à Medicina e na restauração de uma identidade médica para os psiquiatras ${ }^{2}$.

\section{2) Conceitos e objetivos}

De modo geral, a interconsulta é a presença de um médico psiquiatra em uma unidade ou serviço médico geral atendendo à solicitação de um médico de outra especialidade ${ }^{1}$. Ou seja, é uma atividade interprofissional e interdisciplinar. Num sentido mais estrito, podemos definir a interconsulta psiquiátrica e seus objetivos como uma subespecialidade da psiquiatria que $\mathrm{e}^{1,2,4,5}$ :

- atua na interface com a Medicina em geral;

- auxilia na assistência ao paciente de hospital geral;

- colabora na abordagem psicossocial (aspectos psiquiátricos, psicológicos e sociais) do paciente;

- e auxilia na tarefa de ensino e pesquisa.

Partindo dessa definição em quatro itens, os objetivos da interconsulta podem ser definidos assim:

- auxiliar no diagnóstico e no tratamento de pacientes com comorbidade entre doença clínica e doença psiquiátrica;

- auxiliar e instrumentalizar o médico nãopsiquiatra a reconhecer e tratar situações de natureza psiquiátrica;

- auxiliar no entendimento biopsicossocial do paciente e de suas doenças;

- difundir o conhecimento psiquiátrico entre outras áreas da Medicina.

\section{3) Natureza das solicitações de consultoria}

As solicitações de consultoria psiquiátrica num hospital geral variam enormemente. Porém, essa natureza diversificada pode ser reunida em três campos de abordagem ${ }^{1,4}$ :

a) avaliação psiquiátrica clínica;

b) avaliação psicológica e familiar;

c) avaliação de aspectos bioéticos;

A variedade de aspectos envolvidos numa consultoria psiquiátrica freqüentemente exige a participação de uma equipe de profissionais de 
outras áreas da saúde, como Psicologia e Serviço Social. Talvez, num futuro próximo, seja mais correto falarmos em uma equipe de interconsulta psiquiátrica.

\section{Avaliação psiquiátrica clínica}

Embora com algumas variações, a maioria dos dados demonstra que cerca de $30 \%$ dos pacientes internados em unidades clínicas ou cirúrgicas apresentam algum diagnóstico psiquiátrico ${ }^{4}$. Nesse grupo, as evidências atuais apontam para a seguinte prevalência (média) de diagnóstico psiquiátrico: a) transtornos do humor (35\%); b) transtornos de ansiedade $(20 \%)$; c) transtornos relacionados ao uso de substâncias (20\%); transtornos mentais orgânicos (20\%); d) outros (5\%).

$\mathrm{Na}$ avaliação desses dados são necessárias algumas considerações:

a) os transtornos de humor incluem depressão e transtorno bipolar;

b) os transtornos de ansiedade incluem reações de ajustamento e sintomas somatoformes;

c) e os transtornos orgânicos incluem delirium, demência e sintomas psiquiátricos secundários à doença orgânica.

É interessante observar que os estudos epidemiológicos costumam apresentar amplas variações. Assim, por exemplo, uma recente revisão ${ }^{7}$ demonstrou que os transtornos de humor (56\%) e os relacionados ao uso de álcool (29\%) foram os principais diagnósticos efetuados por consultorias psiquiátricas na década de 1990 no University Medical Center da Universidade de Minnesota, nos Estados Unidos. Conforme outro estudo $^{2}$, quando corrigido, principalmente, o viés de idade, a prevalência de transtornos mentais orgânicos chega a até $22 \%$. Porém, esse estudo não cita os problemas relacionados a substâncias. Tal diversidade nos permite algumas reflexões: a) a rotina de solicitação de consultorias flutua muito de serviço para serviço; b) o reconhecimento e conseqüente diagnóstico de alguns transtornos estão se modificando nos últimos anos; c) a coleta de dados e os critérios diagnósticos não apresentam um padrão sistemático. Também vale observar que os transtornos psiquiátricos costumam se apresentar em comorbidade. Podemos, por exemplo, atender um paciente com personalidade borderline que apresenta uma reação de ajustamento com humor depressivo.

Avaliação psicológica e familiar

Considerando que um dos objetivos da consultoria é a abordagem biopsicossocial do paciente, a avaliação dos seus aspectos psicológicos e familiares é fundamental. É de grande importância para o psiquiatra o estabelecimento de um diagnóstico psicodinâmico ${ }^{4}$. Nesse sentido, cabe ao consultor a identificação dos mecanismos de defesa utilizados pelo paciente, do seu funcionamento psicológico usual e da sua estrutura de personalidade.

De igual importância, a avaliação da família possibilita a complementação dos dados coletados junto ao paciente, equipe e prontuário. Além disso, um entendimento do funcionamento familiar e das formas (saudáveis e patológicas) pelas quais a família lida com os problemas do paciente também é necessária para o diagnóstico e o tratamento.

\section{Avaliação bioética}

Algumas vezes, o psiquiatra será chamado para auxiliar a equipe assistente a decidir questões complexas. Não raramente, o pedido de consultoria se relaciona a pacientes que não desejam seguir o tratamento ou que não colaboram com a equipe. Também é freqüente a solicitação para auxiliar na abordagem de pacientes terminais ou que receberam diagnósticos "difíceis", como câncer ou infecção pelo HIV. Nessas situações, nos depararemos com questionamentos relativos, por exemplo, ao grau de autonomia do paciente, ao nível de juízo crítico, e à necessidade de manter ou não o sigilo, entre outros. Para o melhor encaminhamento dessas questões, torna-se muito importante para o psiquiatra ter um bom treinamento em bioética e o assessoramento, sempre que necessário, dos comitês de ética presentes nos hospitais.

Consultoria psiquiátrica: a "situação total"4

A revisão da natureza das consultorias nos leva a refletir sobre o seu aspecto múltiplo. Um mesmo paciente terá sempre os três aspectos comentados. Cabe ao consultor identificá-los e encaminhá-los corretamente. Por exemplo, um paciente que se nega a obedecer as condutas da equipe pode apresentar uma doença psiquiátrica que Ihe imponha prejuízo do juízo crítico e um baixo limiar a frustrações, além de uma família pouco continente. Caberá ao consultor determinar a sua capacidade de autonomia no tratamento. É tarefa do psiquiatra consultor identificar o aspecto preponderante no diagnóstico, as prioridades do encaminhamento e as necessidades da equipe. 


\section{4) Interconsulta psiquiátrica em hospital de trauma}

Semelhanças e diferenças da interconsulta psiquiátrica em hospital de trauma e hospital geral

Em termos gerais, a interconsulta psiquiátrica em hospital de trauma $(\mathrm{HT})$ obedece aos mesmos princípios daquela realizada em um hospital geral. No entanto, algumas particularidades acabam por diferenciar a psiquiatria no HT, conforme exposto a seguir.

O trauma é uma doença resultante de injúria intencional ou não. Pode significar desde uma simples lesão ou machucadura causada por pequenos "acidentes" domésticos até "acidentes" graves ocorridos no trânsito, decorrentes da violência urbana e inter-pessoal, assim como tentativas de suicídio ${ }^{8}$. O trauma é uma doença que vem ganhando importância nas últimas décadas, e, atualmente, é a principal causa de morte entre o primeiro e $44^{\circ}$ ano de vida ${ }^{9}$. O número de anos potencialmente perdidos por trauma é maior do que a soma resultante de doenças cardiovasculares e neoplásicas ${ }^{9}$. Portanto, num hospital de trauma iremos encontrar indivíduos que, além de possíveis antecedentes mórbidos, apresentam uma doença aguda grave e potencialmente fatal.

A noção de "acidente" não é mais aceita no meio médico ${ }^{9}$. A grande maioria dos traumas tem prevenção, e a sua ocorrência resulta da não-observância disso. Assim, a história do trauma pode estar vinculada a transtornos psiquiátricos prévios, já identificados ou não. Para citar alguns exemplos, acidentes de trânsito podem estar vinculados ao abuso de drogas, queimaduras a tentativas de suicídio, agressões a exposição moral, etc. Portanto, a forma como ocorreu o trauma já fornece pistas acerca de um possível transtorno psiquiátrico.

A relação do trauma com os transtornos psiquiátricos é uma "via de mão dupla": o trauma é um fator de risco para transtornos psiquiátricos ${ }^{10}$ e esses, por sua vez, são fatores de risco para a ocorrência de trauma. A nãoidentificação de aspectos psiquiátricos relacionados ao trauma pode contribuir para a sua reincidência ${ }^{10}$. Para ilustrar, um paciente alcoolista pode sofrer um acidente de carro, se recuperar e num outro momento se envolver em violência inter-pessoal.

Por fim, a interconsulta psiquiátrica em hospital de trauma é uma subespecialidade recente dentro da psiquiatria e mesmo da interconsulta. A sua consolidação depende do envolvimento dos médicos psiquiatras na sua divulgação dentro do meio científico, bem como da interação com os profissionais da "linha de frente" do trauma (os médicos cirurgiões e de emergência).

Transtornos psiquiátricos no hospital de trauma

As doenças psiquiátricas podem ser consideradas como fatores de risco para a ocorrência de trauma e sua recidiva ${ }^{10}$. Assim como descrito anteriormente, os dados apresentam ampla variabilidade. Citações na literatura apontam a ocorrência de abuso de drogas entre 20 e $55 \%$ dos pacientes com trauma $^{10}$. No entanto, estudos transversais ${ }^{6}$ citam uma prevalência de $13 \%$ para esse transtorno. O principal viés parece ser o de seleção. Alguns estudos ${ }^{6}$ utilizam como amostra a população de pacientes sob consultoria psiquiátrica, enquanto outros ${ }^{10}$ citam dados gerais, independentes da avaliação psiquiátrica. Além disso, podemos ter um viés de confusão. $O$ registro de um transtorno como sendo o principal pode mascarar a presença de outro. Não há especificações claras de quantos dos pacientes que tentaram o suicídio, por exemplo, apresentavam transtorno de humor e abuso de substâncias, só para citar uma possível interação. É possível que muitos pacientes sejam identificados como abusadores de drogas pela equipe assistente mas não sejam avaliados pelo psiquiatra. Essa impressão é corroborada por um estudo ${ }^{11}$ que cita como prováveis fatores a resistência de alguns profissionais em reconhecer o uso de drogas como doença (e a conseqüente necessidade de avaliação por um especialista), a ausência de programas de tratamento nos hospitais de trauma e a falta de exames de triagem para drogas no ambiente de emergência (ou a ausência de rotina para isso).

Outro dado esclarecedor vem de um estudo transversal ${ }^{10}$ que demonstrou prevalência de transtornos psiquiátricos em $29 \%$ dos pacientes traumatizados. Desses, cerca de $27 \%$ obtiveram o diagnóstico de abuso de drogas e 1,3\% receberam o diagnóstico de depressão. Nesse estudo, o diagnóstico foi realizado pela equipe assistente, e não por psiquiatras. A discrepância entre $29 \%$ de diagnóstico psiquiátrico pela equipe assistente e $10 \%$ de solicitações de consultoria ${ }^{11}$ (ponto discutido mais adiante) ajuda a entender as diferenças nos achados epidemiológicos.

As evidências da literatura, em estudos 
transversais, sugerem uma associação entre sintomas psiquiátricos e trauma. A avaliação psiquiátrica é solicitada em cerca de $10 \%$ dos pacientes hospitalizados por trauma ${ }^{6}$. Esse grupo costuma apresentar um tempo de internação duas vezes maior em relação ao grupo não-referenciado. Esse prolongamento da internação não parece estar relacionado com a severidade do trauma, que costuma ser discretamente menor nos pacientes avaliados pelo psiquiatra. As possíveis razões incluem a necessidade de esclarecimento diagnóstico, que costuma ser mais demorado na psiquiatria, bem como a necessidade de transferência para uma unidade especializada ${ }^{6}$. A demora decorrente da avaliação de um outro especialista pode contribuir para a nãosolicitação de consultoria pela equipe assistente. Dos pacientes em avaliação psiquiátrica, alguns dados devem ser destacados: a) a tentativa de suicídio (reconhecida pela equipe assistente) é a principal razão de solicitação de consultoria (45\%) (tabela 1); os transtornos de humor são o principal diagnóstico (28\%) (tabela 2); os transtornos de humor são, também, o principal diagnóstico prévio (28\%).

A história psiquiátrica prévia freqüentemente indica a presença de um transtorno mental. Esse achado sugere que o trauma pode ser muito mais a conseqüência de um transtorno psiquiátrico do que a sua causa ${ }^{6}$.

1) Tentativa de suicídio

A tentativa de suicídio (TS) é o principal motivo de solicitação de consultoria em hospital de trauma ${ }^{6,12}$. A tentativa de suicídio é responsável também pela solicitação precoce de consultoria, quando comparada com outros motivos. A TS costuma dispender mais tempo de avaliação do que os outros transtornos. As TS representam 19\% dos traumas violentos (ferimentos por arma de fogo, queimaduras, quedas de altura, etc.), embora somente $10 \%$ dos traumas sejam avaliados pelo psiquiatra ${ }^{6}$.

Um estudo ${ }^{12}$ realizado em hospital geral mostrou que cerca de $45 \%$ dos casos de TS apresentavam comorbidade com abuso de substâncias psicoativas (SPA). Nessa grupo, a comorbidade mais comum foi entre depressão e o abuso de substâncias (37\%). Esses indivíduos eram mais freqüentemente homens com idade entre 30 e 60 anos. No entanto, houve uma alta prevalência de TS e abuso de substâncias, independente do sexo e da idade ${ }^{12}$. Além disso, a comorbidade entre depressão e abuso de substâncias foi responsável por escores maiores de ideação suicida (alto risco de suicídio). Isso sugere que a instabilidade de humor no contexto de uso de SPA é um fator de risco para tentativa grave de suicídio ${ }^{12}$.

A TS é um evento que exige uma investigação diagnóstica para evento desencadeante e risco de recidiva. Em hospitais de trauma, a TS costuma ser violenta, o que por si só já é um fator de risco para recidiva ${ }^{13}$.

2) Abuso de substâncias

O abuso de substâncias, em especial o álcool, está relacionado com a ocorrência de trauma ${ }^{10}$. Conforme citado anteriormente, entre 20 e 55\% dos paciente hospitalizados por

Tabela 1 - Solicitações de consultoria em hospital de trauma

\begin{tabular}{ll}
\hline Motivo & $\%$ \\
\hline Tentativa de suicídio & 45 \\
Depressão & 23 \\
Estresse pós-traumático & 8 \\
Doença psiquiátrica prévia & 8 \\
Problemas de conduta & 7 \\
Sintomas conversivos & 3 \\
Outros & 5 \\
\hline
\end{tabular}

Adaptado de Posel \& Moss ${ }^{6}$. 
Tabela 2 - Diagnóstico psiquiátrico em pacientes traumatizados

\begin{tabular}{lc}
\hline Diagnóstico & $\%$ \\
\hline Transtorno de humor & 28 \\
Traumatismo cranioencefálico & 25 \\
Abuso de substâncias & 13 \\
Transtorno de ajustamento & 12 \\
Transtorno de personalidade & 12 \\
Transtorno psicótico & 12 \\
Transtorno de ansiedade & 10 \\
Delirium & 10 \\
Outros & 5 \\
Nenhum & 12 \\
\hline
\end{tabular}

Adaptado de Posel \& Moss 6 .

trauma têm história atual ou prévia de abuso de álcool. Esses pacientes costumam ter risco aumentado de recidiva em trauma e intervenção cirúrgica ${ }^{14}$.

Na unidade de trauma, a equipe assistente costuma reconhecer abuso de álcool ou substâncias em cerca de $27 \%$ dos pacientes ${ }^{10}$. Esses dados foram encontrados também em um estudo que observou intoxicação aguda por álcool em $29 \%$ dos pacientes traumatizados ${ }^{11}$. Apesar do diagnóstico, muitos pacientes não são avaliados pelo psiquiatra. Num estudo citado anteriormente, cerca de $10 \%$ dos pacientes traumatizados são avaliados pelo psiquiatra ${ }^{6}$; desses $17 \%$ recebem o diagnóstico de abuso de substâncias.

O paciente alcoolista traumatizado costuma ser homem, com 40 anos ou menos, usuário de outras drogas, e fica internado menos tempo do que outros pacientes com trauma de igual severidade ${ }^{11}$. Outro estudo ${ }^{10}$ aponta que a duração da internação é 10\% menor em pacientes abusadores de álcool, com um custo $12 \%$ inferior do que para pacientes não-alcoolistas.

O abuso de álcool ou outras drogas está presente em cerca de $20 \%$ dos pacientes traumatizados por queimaduras ${ }^{15}$.

Algumas considerações são pertinentes em relação ao uso de álcool e trauma. A prevalência, ao longo da vida, de abuso de álcool na população em geral é de $13,8 \%{ }^{11}$. A comorbidade entre abuso de substâncias e outros transtornos psiquiátricos costuma ser alta. Dentre os transtornos psiquiátricos, os transtornos de humor têm uma prevalência de comorbidade de $77,2 \%$, constituindo-se na associação mais freqüente com abuso de álcool ${ }^{12}$. Os dados da literatura mostram que os transtornos de humor são os mais diagnosticados em pacientes com trauma. Portanto, a investigação do abuso de álcool é obrigatória na avaliação do paciente traumatizado.

3) Paciente queimado

Conforme citado num estudo recente ${ }^{15}$, a maioria dos pacientes traumatizados por queimaduras apresenta importantes fatores predisponentes. Cerca de $59 \%$ das mulheres e $38 \%$ dos homens apresentam fatores de risco para queimaduras graves. Dentre esses fatores se destacam o abuso de álcool ou drogas, demência, esquizofrenia e transtorno bipolar. Nos casos psiquiátricos, as queimaduras costumam ser secundárias a auto-imolação, juízo crítico prejudicado ou coordenação motora debilitada por intoxicação por substância e comportamento de risco.

Entre os pacientes que tentam o suicídio por auto-imolação, cerca de $70 \%$ têm história psiquiátrica prévia e $55 \%$ já tentaram o suicídio anteriormente ${ }^{16}$. 
A história psiquiátrica também é fator de risco para mortalidade por queimadura. Transtornos de personalidade, tentativa de suicídio, esquizofrenia e abuso de álcool aumentam a mortalidade de pacientes queimados ${ }^{15}$.

No paciente queimado, alguns transtornos psiquiátricos são mais comuns em cada fase do tratamento (tabela 3). Na fase aguda, podemos encontrar delirium por abstinência de drogas, delirium do queimado (burn delirium) e transtorno de estresse agudo. A presença de delirium costuma ser um indicador de pior prognóstico. Algumas reações de ajustamento, como negação, revolta e hostilidade também são comuns nessa fase.

$\mathrm{Na}$ fase reconstrutiva, são comuns os sentimentos de tristeza e luto pela imagem corporal. Nesse período, transtornos como estresse pós-traumático e transtorno de ajustamento e de humor são freqüentes.

4) Traumatismo cranioencefálico (TCE)

O TCE é um evento comum no trauma. A lesão cerebral resultante do TCE provoca uma gama de sintomas neuropsiquiátricos. Uma revisão recente ${ }^{17}$ propõe o termo "seqüela neuropsiquiátrica do TCE”. Tal definição serviria para evitar os termos confusos normalmente utilizados, como síndrome pós-concussional e transtorno mental decorrente de TCE, por exemplo. Nessa revisão utilizaremos o termo síndrome neuropsiquiátrica associada ao TCE (SNP-TCE), uma vez que o termo seqüela pode dar a impressão equivocada de irreversibilidade. As síndromes associadas ao TCE são o déficit cognitivo, distúrbios do humor, ansiedade, psicose, apatia e alterações de comportamento ${ }^{17}$ (tabela 4).

Os principais fatores de risco para a (SNP-
TCE) são idade avançada, arteriosclerose e alcoolismo ${ }^{17}$. Esses fatores costumam retardar processos regenerativos no sistema nervoso central $(\mathrm{SNC})^{18}$. Aspectos da personalidade prévia são decisivos na recuperação do paciente. Como diz Symonds, a reação ao "TCE depende do tipo da cabeça traumatizada"19. Portanto, transtornos de personalidade também são fatores de risco para SNP-TCE. Outros fatores, comuns em doenças psiquiátricas, favorecem o aparecimento da síndrome. São eles: instabilidade conjugal, rede sócio-familiar ausente, desemprego e instabilidade financeira ${ }^{20}$.

\section{1) SNP-TCE: déficit cognitivo}

O TCE costuma causar uma pletora de sintomas cognitivos. Esses sintomas incluem alterações na atenção, vigilância, concentração, memória, linguagem e função executiva. O déficit de memória pode ser verbal ou nãoverbal. Distúrbios no funcionamento executivo incluem déficit no planejamento, organização, seqüenciamento e mudanças das ações, associado a prejuízo no juízo crítico e controle de impulsos ${ }^{17}$.

O tipo de déficit apresentado costuma estar relacionado com a fase do $\mathrm{TCE}^{21}$. A primeira fase é a de perda da consciência que ocorre logo após o TCE. A segunda é caracterizada por um misto de alterações cognitivas e comportamentais como agitação psicomotora, desorientação e desatenção. As duas primeiras fases têm uma duração de até um mês e costumam ser denominadas delirium póstraumático ${ }^{22}$. A terceira fase tem uma duração de 6 a 12 meses e se caracteriza por uma rápida recuperação das capacidades cognitivas prejudicadas anteriormente, atingindo, porém, um platô de recuperação que se sustenta por

Tabela 3 - Transtornos psiquiátricos mais comuns em pacientes queimados

\begin{tabular}{ll}
\hline Fase & Transtorno psiquiátrico \\
\hline Aguda & $\begin{array}{l}\text { Delirium decorrente de } \\
\text { - Abstinência de drogas } \\
\text { - Queimadura } \\
\text { Transtorno de estresse agudo }\end{array}$ \\
& Transtorno de estresse pós-traumático \\
Reconstrutiva & Transtorno de ajustamento \\
& Transtorno de humor \\
\hline
\end{tabular}

Adaptado de llechukwu ${ }^{15}$. 
Tabela 4 - Síndromes neuropsiquiátricas associada a TCE

1. Déficit cognitivo
2. Distúrbios de humor
- Depressão
- Mania
3. Distúrbios de ansiedade
4. Psicose
5. Apatia
6. Distúrbio comportamental
- Variante maior
- Variante menor
7. Outros
- Alterações de sono
- Cefaléia

Adaptado de Rao \& Lyketsos ${ }^{17}$.

até 24 meses após o TCE. A quarta fase é caracterizada pelas seqüelas cognitivas permanentes e inclui lentificação no curso do pensamento, déficit na atenção, memória, linguagem e função executiva, bem como pensamento concreto. Tal fase é comumente descrita como demência pós-TCE ${ }^{17}$.

4.2) SNP-TCE: transtornos de humor

Síndrome depressiva maior ocorre em cerca de $25 \%$ dos pacientes com TCE $^{23}$. Sentimentos de perda, desmoralização e desesperança costumam ser observados precocemente e acompanhados por sintomas de disforia. Fadiga, irritabilidade, ideação suicida, anedonia e insônia são proeminentes entre o sexto e o $24^{\circ}$ mês posterior ao TCE ${ }^{24}$. Estudos demonstram que um funcionamento psicossocial pobre e história de doença psiquiátrica são os principais fatores de risco para depressão pós-TCE ${ }^{23}$. A presença de lesão frontolateral esquerda e gânglios da base esquerda está associada a maior probabilidade de desenvolvimento de depressão ${ }^{17}$.

O desenvolvimento de mania pós-TCE é menos comum do que o desenvolvimento de depressão, mas bem mais freqüente do que na população em geral. No TCE, a prevalência de mania é de $9 \%{ }^{25}$. Os principais fatores de risco são história familiar de transtorno de humor e atrofia subcortical prévia ${ }^{17}$. A mania é mais comum em pacientes com lesões em estruturas límbicas do hemisfério direito ${ }^{25}$.

4.3) Distúrbios de ansiedade

O paciente pós-TCE pode apresentar uma variante de todos os transtornos de ansiedade.
Mais comumente, encontra-se um quadro de ansiedade persistente e flutuante associado com preocupação excessiva e incontrolável, característico de ansiedade generalizada. Nesse caso, os distúrbios de ansiedade estão mais associados a lesões no hemisfério direito ${ }^{17}$.

4.4) Psicose

Até $10 \%$ dos pacientes pós-TCE podem desenvolver um quadro psicótico esquizofreniforme ${ }^{17}$. A maioria desses pacientes não tem história familiar de esquizofrenia. Em pacientes com diagnóstico prévio de esquizofrenia, a exacerbação dos sintomas ocorre em cerca de $15 \%$ dos casos. A apresentação dos sintomas pode ser aguda ou crônica, transitória ou persistente, e pode ou não estar associada a alterações de humor. Não há uma relação evidente entre surgimento de sintomas psicóticos e região do cérebro traumatizada.

\section{5) Apatia}

Cerca de $10 \%$ dos pacientes pós-TCE podem apresentar um quadro de apatia, sem apresentar critérios para depressão ${ }^{17}$. Tal quadro é caracterizado por uma síndrome de desinteresse, inércia, falta de motivação e embotamento afetivo, sem alterações cognitivas. Essa síndrome parece estar relacionada a lesões no lobo frontal ${ }^{26}$.

\section{6) Distúrbio comportamental}

Uma grande parte dos pacientes pós-TCE costuma apresentar uma síndrome com alterações mistas na cognição, humor e 
comportamento ${ }^{27}$. Uma recente revisão propõe o termo distúrbio comportamental para descrever essa manifestação ${ }^{17}$. Tal síndrome é dividida em duas variantes: maior e menor.

A variante maior (tabela 5) pode se relacionar a uma lesão focal ou difusa. Lesões na área fronto-orbital causam desinibição, enquanto que as lesões na convexidade dorsal do lobo frontal podem causar disfunção executiva ${ }^{26}$. Lesões no lobo temporal causam labilidade emocional e déficit de memória.

A variante menor (tabela 6), comumente chamada de síndrome pós-concussional, é a entidade neuropsiquiátrica mais diagnosticada no pós-TCE ${ }^{17}$. Trata-se de um conjunto de sinais e sintomas visto com freqüência em traumatismos de gravidade leve-moderada, mas podendo ocorrer em qualquer caso. É a síndrome mais comum em casos de TCE sem perda da consciência. A maioria dos pacientes se recupera dentro de seis meses pós-TCE, mas alguns sintomas podem persistir por até um ano. Cerca de 15\% apresentam sintomas por tempo indefinido. Supõe-se que uma lesão axonal difusa, secundária ao trauma da aceleração e desaceleração, seja responsável pelo aparecimento desse problema. O exame neurológico e de imagem costuma ser normal. Estudos com tomografia por emissão de pósitrons (PET) e tomografia por emissão de fóton único (SPECT) demonstram alterações focais e inespecíficas no metabolismo da glicose e fluxo sangüíneo cerebral ${ }^{17}$.

\section{CONCLUSÃO}

A importância da interconsulta psiquiátrica no hospital geral parece estar consolidada. Um movimento de mais de um século vem criando uma identidade hospitalar e médica para a psiquiatria. Acompanhando essas modificações, a psiquiatria passou a se ocupar também de uma outra área da Medicina hospitalar: o trauma. Essa doença dos tempos modernos, de custos imensos e grande impacto na saúde pública, tem exigido atenção

Tabela 5 - Distúrbio comportamental, variante maior

\begin{tabular}{lcc}
\hline Humor & Cognição & Comportamento \\
\hline Irritabilidade & Déficit na atenção & Impulsividade \\
Raiva & Déficit na memória & Agressividade \\
Medo & Déficit de função executiva & Hiperatividade \\
& Prejuízo no juízo crítico & Hiperfagia \\
& & Síndrome de PICA \\
\hline
\end{tabular}

Adaptado de Rao \& Lyketsos ${ }^{17}$.

Tabela 6 - Distúrbio comportamental, variante menor

\begin{tabular}{lcc}
\hline Humor & Cognição & Comportamento \\
\hline Depressão & Déficit de memória & Cefaléia \\
Ansiedade & Atenção diminuída & Náuseas \\
Irritabilidade & Concentração diminuída & Tontura / vertigem \\
& Disfunção executiva leve & Diplopia \\
& Insônia \\
& Surdez / zumbidos \\
& Maior sensibilidade a luz/barulhos \\
& Fadiga \\
& Menor coordenação motora \\
\hline
\end{tabular}


crescente da psiquiatria. Para citar alguns exemplos, em Porto Alegre, dois grandes hospitais de trauma contam com equipes de consultoria psiquiátrica; são eles o Hospital de Pronto-Socorro (HPS) e o Hospital Cristo Redentor (HCR).

O psiquiatra de trauma necessita de um sólido conhecimento dos princípios gerais da interconsulta psiquiátrica. Além disso, precisa se abastecer de informações e ensinamentos específicos sobre a interface traumapsiquiatria. A proposta deste trabalho foi justamente abordar os aspectos gerais dessa questão. Procura-se assim, iniciar uma discussão sobre o que, exatamente, é a interconsulta psiquiátrica em hospital de trauma. Para isso, buscou-se estabelecer as intersecções e diferenças dessa modalidade em relação à interconsulta geral: a principal diferença se refere à especificidade da doença trauma. Foi observado também que a apresentação, a prevalência e o raciocínio clínico apresentam particularidades nos casos psiquiátricos de trauma. Torna-se fundamental para o médico psiquiatra consultor ter em mente as relações entre os diferentes tipos de trauma e a sua relação com possíveis transtornos psiquiátricos prévios, assim como as prováveis complicações psiquiátricas decorrentes do trauma. Por fim, foram enfatizadas as conseqüências neuropsiquiátricas do traumatismo cranioencefálico. Esta é, ainda mais do que as outras, uma categoria específica da psiquiatria de trauma.

O presente trabalho procurou fazer uma abordagem geral da interconsulta psiquiátrica em hospital de trauma através de uma revisão não-sistemática da literatura. Esse fato limita a capacidade de generalização da presente revisão. No entanto, conforme discutido ao longo do texto, as amplas variações de metodologia nos trabalhos já publicados dificultam a sistematização dos dados e apontam para a necessidade de se buscar uma homogeneidade na pesquisa da interconsulta psiquiátrica em hospital de trauma.

Dessa maneira, as evidências atuais indicam que devemos trabalhar no desenvolvimento (ou adaptação) de escalas diagnósticas, sistematização da coleta de dados e estabelecimento de estudos controlados. Os psiquiatras que trabalham nessa área se encontram frente ao desafio de gerar novos conhecimentos sobre a epidemiologia, a clínica e o tratamento dos transtornos psiquiátricos associados ao trauma.

\section{REFERÊNCIAS BIBLIOGRÁFICAS}

1. Nogueira-Martins LA. A interconsulta como instrumento da psiquiatria de hospital geral. Cad IPUB. 1997;6:33-44.

2. Kaplan H, Sadock BJ. Tratado de psiquiatria. $6^{\underline{a}}$ ed. Porto Alegre: Artmed; 1999. vol. 2.

3. Brasil. Lei Federal $n^{\circ} 10216$, de 6 de abril de 2001. Brasília, 2001.

4. Sandri A, Bertuol CS, Conte C, et al. Interconsulta psiquiátrica no hospital geral: diagnóstico da situação total. Rev Psiquiatr RS. 2000;22(2):138-47.

5. Westphal JR, Freeman AM. Consultation-liason psychiatry. In: Ebert MH, Loosen PT, Nurcombe B. Current diagnosis and treatment in psychiatry. St. Louis: Lange Medical Books/McGraw-Hill; 2000. p. 163-79.

6. Posel C, Moss J. Psychiatry morbidity in a series of patients referred from a trauma service. Gen Hosp Psychiatry. 1998;20:198-201.

7. Grant JE, Meller W, Urevig B. Changes in psychiatry consultation over ten years. Gen Hosp Psychiatry. 2001;23:261-5

8. Montovani. Disponível em: http://www.hc.unicamp.br/ especialidades_medicas/ trauma/sobre.htm. Acessado em 2003.

9. American College of Surgeons. Advanced trauma life support (ATLS). Chicago: American College of Surgeons; 1996.

10. Zatzick DF, Kang SM, Kim SY, Leigh P, Kravitz R, Drake $\mathrm{C}$, et al. Patients with recognized psychiatric disorders in trauma surgery: incidence, inpatient length of stay and cost. J Trauma. 2000;49:487-95.

11. Blondell RD, Looney SW, Krieg CL, Spain DA. A comparison of alcohol-positive and alcohol negative trauma patients. J Stud Alcohol. 2002;63:380-3.

12. Dhossche DM, Meloukheia AM, Chakravorty S. The association of suicide attempts and comorbid depression and substance abuse in psychiatry consultation patients. Gen Hosp Psychiatry. 2000;22:281-8.

13. Schmitt R, Chachamovich E, Kapczinski F. Risco de suicídio: avaliação e manejo. In: Kapczinki F, Quevedo J, Schmitt R, Chachamovich E. Emergências psiquiátricas. Porto Alegre: Artmed; 2001. p. 149-65.

14. Rivara FP, Koespell TD, Jurkovich GJ, Gurney JG, Soderberg $R$. The effects of alcohol abuse on readmission for trauma. JAMA. 1993;270:1962-4.

15. Ilechukwu ST. Psychiatry of the medically ill in the burn unit. Pshychiatr Clin North Am. 2002;25(1):129-47.

16. Sonneborn CK, Vanstraelen PM. A retrospective study of self inflicted burns. Gen Hosp Psychiatry. 1992;14:404-7.

17. Rao V, Lyketsos C. Neuropsychiatry sequelae of traumatic brain injury. Psychosomatics. 2000;41:95-103.

18. Lishman WA. Physiogenesis and psychogenesis in the post-concussional syndrome. $\mathrm{Br} J$ Psychiatry. 1988;153:460-9.

19. Symonds CP. Mental disorder following head injury. Proceedings of the Royal Society of Medicine. 1937;30:1081-92.

20. Whitlock FA, Stoll JK, Rekhdahl RJ. Crisis, life events, and accidents. Aust N Z J Psychiatry. 1977;11:127-32.

21. Levin HS. Neurobehavioral sequelae of head injury. In: Cooper PR, editor. Head injury. 2nd ed. Baltimore: Williams \& Wilkins; 1987.

22. Kwentus JA, Hart RP, Peck ET, Kornstein S. Psychiatric complications of closed head trauma. Psychosomatics. 1985;26:8-15.

23. Fedoroff JP, Starkstein SE, Forrester AW, Geisler FH, Jorge RE, Arndt SV, et al. Depression in patients with traumatic brain injury. Am J Psychiatry. 1992;149:91823. 
24. Hinkeldey NS, Corrigan JD. The structure of head-injured patients' neurobehavioural complaints: a preliminary study. Brain Inj. 1990;4:115-33.

25. Jorge RE, Robinson RG, Starkstein SE, Arndt SV Forrester AW, Geisler FH. Secondary mania following traumatic brain injury. Am J Psychiatry. 1993;150:91621.

26. Duffy JD, Campbell JJ. The regional prefrontal syndromes: a theoretical and clinical overview. J Neuropsychiatry Clin Neurosci. 1994;6:379-87.

27. Gerring JP. Psychiatric sequelae of severe closed head injury. Pediatr Rev. 1986;8:115-21.

\section{RESUMO}

Os autores se propõem a abordar os aspectos particulares da interconsulta psiquiátrica em um hospital de trauma. Para isso, revisam os aspectos gerais de uma interconsulta em um hospital geral, e, posteriormente, destacam as características que diferenciam a consultoria psiquiátrica em um hospital de trauma. Os eventos psiquiátricos mais relevantes no trauma são as tentativas de suicídio violentas, o abuso de substâncias e as reações psíquicas ao traumatismo. O ponto de destaque se refere à especificidade da relação entre trauma e doenças psiquiátricas: ambos podem ser causa ou conseqüência. A interconsulta psiquiátrica é um novo campo de atuação interdisciplinar e de produção científica para a psiquiatria.

Descritores: Interconsulta, trauma, hospital, psiquiatria.

\section{ABSTRACT}

The objective of this paper is to describe the main aspects of consultation-liaison psychiatry at a trauma hospital. The characteristics of consultationliaison psychiatry in general hospitals are discussed. After that, the unique characteristics of consultationliasion psychiatry at trauma hospitals are presented. The most common psychiatric trauma-related events are violent suicide attempts, substance abuse and psychological reaction to trauma. The main focus is on the specificity of the relationship between trauma and psychiatric disorders: they can be both a consequence and a cause. This is a new field for interdisciplinary action and scientific research in Psychiatry.

Keywords: Consultation-liaison, trauma, hospital, psychiatry.

Title: Aspects of consultation-liaison psychiatry at trauma hospitals

\section{RESUMEN}

Los autores se proponen a abordar aspectos particulares de la interconsulta psiquiátrica en un hospital de trauma. Para eso, revisan los aspectos generales de una interconsulta en hospital general y, posteriormente, destacan las características que diferencian la consultoría psiquiátrica en un hospital de trauma. Los eventos psiquiátricos más relevantes en el trauma son las tentativas de suicidio violentas, el abuso de substancias y las reacciones psíquicas al traumatismo. El destaque se refiere a la especificidad de la relación del trauma y las enfermedades psiquiátricas: ambos pueden ser causa 0 consecuencia. Ese es un nuevo campo de actuación interdisciplinaria y producción científica para la psiquiatría.

Palabras clave: Interconsulta, trauma, psiquiatría, hospital.

Título: Aspectos de la interconsulta psiquiátrica en hospital de trauma

Correspondência:

Ricardo Schmitt

Rua Guaporé, 33-D/401

CEP 89802-300 - Chapecó - SC

Fone: (49) 329.4961

E-mail: schmitt@superip.com.br

Copyright (C) Revista de Psiquiatria do Rio Grande do Sul - SPRS 